\title{
Primary Microcephaly
}

Harold Chen*

Perinatal and Clinical Genetics, Department of Pediatrics, LSU Health Sciences Center, Shreveport, LA, USA

Primary microcephaly $(\mathrm{MCPH})$ is a rare autosomal recessive neurodevelopmental disorder characterized by two principal features, microcephaly present at birth and nonprogressive mental retardation (Woods et al. 2005). The incidence is about 1 in 10,000 in consanguineous populations (Thornton and Woods 2009), less in non-consanguineous populations (between 1 in 30,000 and 1 in 2 million).

\section{Synonyms and Related Disorders}

Autosomal recessive primary microcephaly; Premature chromosome condensation (PCC) syn drome; Premature chromosome condensation with microcephaly and mental retardation syndrome

\section{Genetics/Basic Defects}

1. Inheritance; autosomal recessive

2. Cytogenetic features (Neitzel et al. 2002)

(a) Premature chromosome condensation in G2 phase associated with autosomal recessive microcephaly and mental retardation in two siblings from consanguineous parents

(b) A high frequency of prophase-like cells $(>10 \%)$ in lymphocytes, fibroblasts, and lymphoblast cell lines with an otherwise normal karyotype

3. Molecular genetics (Kaindl et al. 2010; Mahmood et al. 2011)

(a) MCPH1

(i) Chromosome: $8 \mathrm{p} 23.1$

(ii) Gene: $\mathrm{MCPH1}$ (microcephalin)

(iii) Cellular location: nucleus/chromatin

(iv) Proposed function: DNA damage repair, chromosome condensation

(b) $\mathrm{MCPH} 2$

(i) Chromosome: 19q13.12-q13.2

(ii) Gene: WDR62 (WD repeat-containing protein 62)

(iii) Cellular location: nucleus, centrosomal during mitosis

(iv) Proposed function: not yet established (expression pattern resembles with ASPM)

(c) $\mathrm{MCPH} 3$

(i) Chromosome: $9 \mathrm{q} 33.2$

(ii) Gene: $C D K S R A P 2$ (cyclin-dependent kinase 5 regulatory associated protein 2)

(iii) Cellular location: centrosome/midbody

(iv) Proposed function: regulation of microtubule function/centrosome maturation

(d) $\mathrm{MCPH} 4$

\footnotetext{
*Email: hchen@1suhsc.edu
} 

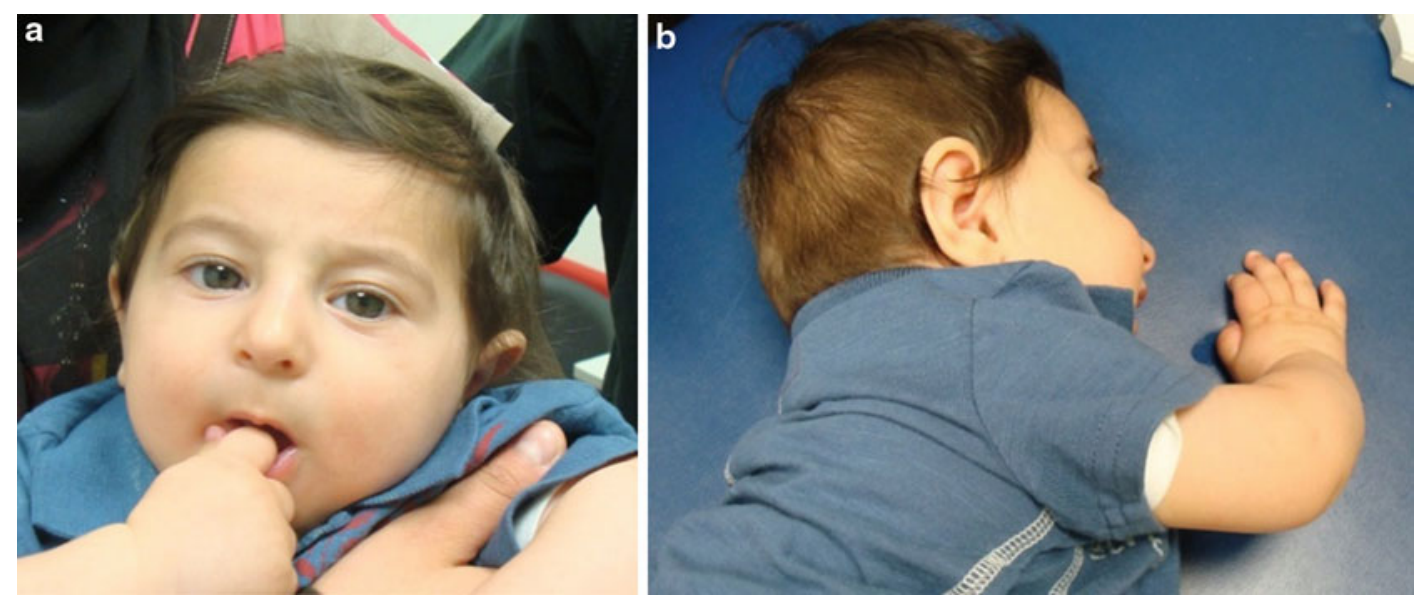

Fig. 1 (a, b) An 8-month-old Arabic boy was evaluated for developmental delay and microcephaly. Her older sister who expired at 15 months of age had microcephaly, developmental delay, and hypotonia. Normal laboratory tests include chromosome microarray analysis, chromosome analysis, plasma amino acids, urine organic acids, acylcarnitine profile, and VLCFA for peroxisomal disorders. Since two siblings are similarly affected in a family, the disorder most likely represents an autosomal recessive disorder of primary microcephaly
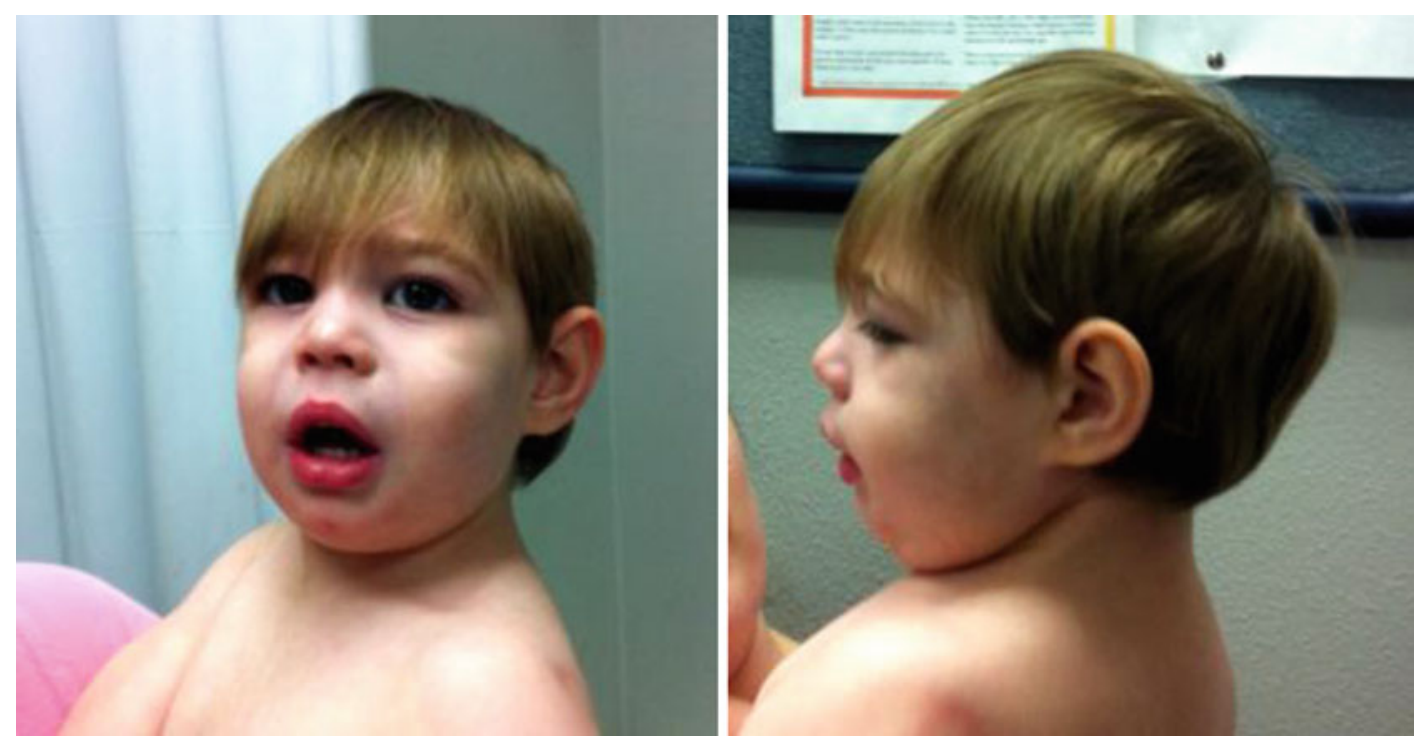

Fig. 2 (a, b) This 3-year-old Caucasian boy was evaluated for primary microcephaly. He had slightly sloping forehead with a very small cranial vault, slightly prominent ears, and mild retromicrognathia. He was born after 40 weeks gestation. It was a normal spontaneous delivery. The birth weight was 3,180 g, birth length $45.9 \mathrm{~cm}$, and HC $29.5 \mathrm{~cm}$ (-3 standard deviations). TORCH titers were negative. He had unremarkable 3D CT. MRI of the brain showed a significantly decreased cranial to facial proportion with a sloping forehead and flattened occiput, abnormal cortical gyral/sulcal development with unusually broad and shallow gyri, and simplified sulcal pattern with generalized prominence of the extra-axial CSF space and thickening of the cortical mantle. The corpus callosum was small. The pituitary and midline structures were normal. This findings are compatible with group I primary microcephaly with an abnormal simplified gyral pattern, but with normal myelination. Craniosynostosis was not present. He has a history of seizures. Currently, he has developmental delay and receives speech and occupational therapy (Courtesy of Dr. Susanne Ursin) 
a

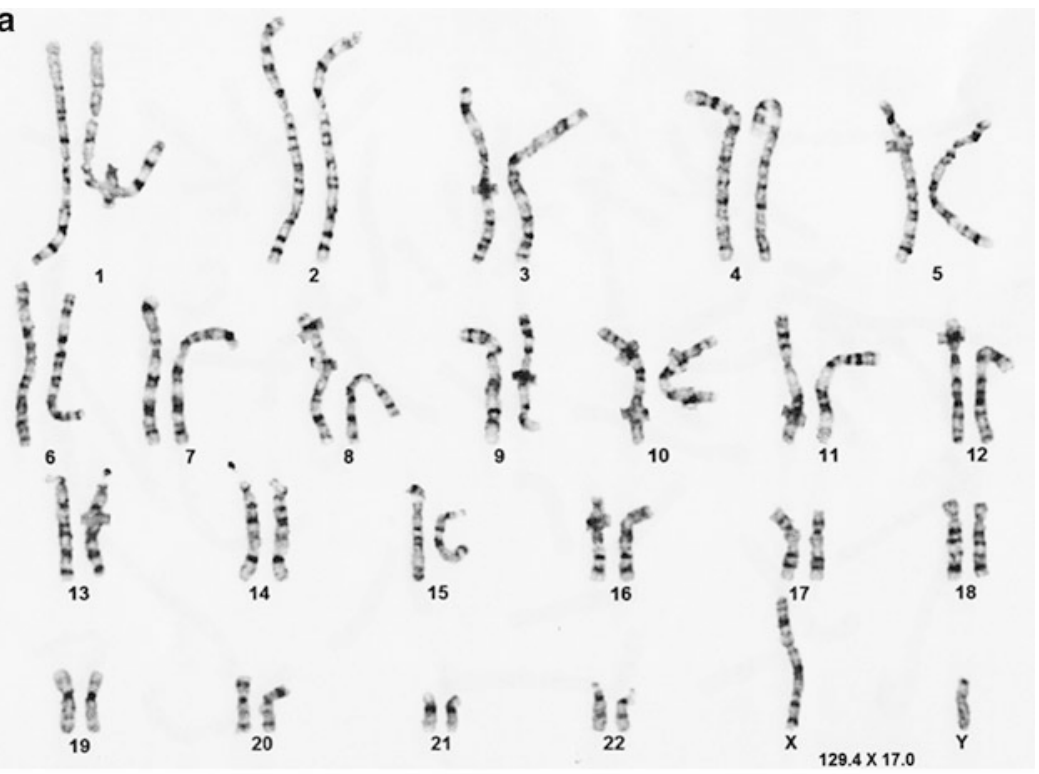

b

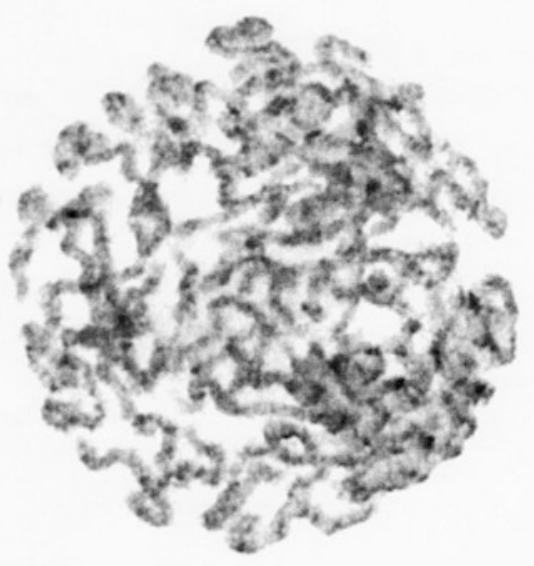

Fig. 3 (a, b) High-resolution cytogenetic analysis of metaphase cells from blood lymphocytes showed a cytogenetic male (a). However, approximately $14 \%$ of cells (normal reference range of $<2 \%$ showed prophase-like cells) (b). Increased number of prophase-like cells has been reported in primary microcephaly in the literature. $M C P H 1$ gene sequencing did not identify any mutation in the $M C P H 1$ gene. However, the diagnosis cannot be completely ruled out due to mutations not detected by this assay or mutations in another gene. The frequency of mutations in the $M C P H 1$ gene ranges from $3.4 \%$ to $8.25 \%$ of the population affected with primary microcephaly (Courtesy of Dr. Leonard Prouty)

(i) Chromosome: $15 \mathrm{q} 15.1$

(ii) Gene: CASC5 (cancer susceptibility candidate 5)

(iii) Cellular location: kinetocore

(iv) Proposed function: kinetochore integrity

(e) MCPH5

(i) Chromosome: 1q31.3

(ii) Gene: ASPM (abnormal spindle-like, microcephaly)

(iii) Cellular location: pericentrosomal/ midbody

(iv) Proposed function: orientation of mitotic spindles during embryonic neurogenesis 
(f) MCPH6

(i) Chromosome: $13 \mathrm{q} 12.12$

(ii) Gene: CENPJ (centromeric protein J)

(iii) Cellular location: centrosome/midbody

(iv) Proposed function: centriole length control/microtubule function

(g) $\mathrm{MCPH} 7$

(i) Chromosome: $1 \mathrm{p} 33$

(ii) Gene: STIL (SCL/TAL1-interrupting locus)

(iii) Cellular location: pericentrosomal

(iv) Proposed function: spindle organization/cell cycle progression

(h) MCPH8 (Hussain et al. 2012)

(i) Chromosome: $4 \mathrm{q} 12$

(ii) Gene: CEP135

(iii) Cellular location: centrosome

(iv) Proposed function: disorganized microtubule networks and mutant protein not localized to the centrosome

(i) MCPH9 (Genin et al. 2012)

(i) Chromosome: $15 \mathrm{q} 21.1$

(ii) Gene: CEP152 (centrosomal protein 152)

(iii) Cellular location: centrosome

(iv) Proposed function: involved in centriole duplication

(j) MCPH10 (Yang et al. 2012)

(i) Chromosome: 20q13.12

(ii) Gene: ZNF335 (zinc finger protein 335)

(iii) Cellular location: neuronal cells

(iv) Proposed function: disrupts the proliferation and proper differentiation of neuronal cells

(k) MCHP11 (Awad et al. 2013)

(i) Chromosome: 12p13.31

(ii) Gene: $\mathrm{PHCl}$ (polyhomeotic-like 1)

(iii) Cellular location: chromatin

(iv) Proposed function: abnormalities in chromatin regulation

\section{Clinical Features}

1. Main features (Woods et al. 2005; Mahmood et al. 2011)
(a) Microcephaly (2 SD below the mean) at birth, further "relative" reduction of head circum- ference in the first years of life
(b) Reduction in cerebral cortex volume
(c) Simplification of gyral pattern
(d) Mild to severe nonprogressive mental retardation (borderline IQ possible)

2. Inconsistent features

(a) Neurological/neuropsychological
(i) Seizures (tonic-clonic, focal, generalized): very rare but cannot exclude the diagnosis (ASPM)

(ii) Delay in early motor milestone, especially speech delay 
(iii) Behavior pattern: sometimes aggressive but otherwise have a happy effect and easy to handle

(iv) Sleep disorder

(b) Other features

(i) Sloping forehead common but is not always associated

(ii) Short stature in some cases $(M C H P 1)$

(iii) Early puberty

3. Differential diagnosis

(a) Nongenetic and genetic causes of "primary microcephaly with mental retardation," e.g.,

(i) Congenital infection with toxoplasma

(ii) Maternal alcohol overconsumption during pregnancy

(iii) Rubinstein-Taybi syndrome

(b) Secondary microcephaly: indicates a progressive neurodegenerative condition

\section{Diagnostic Investigations}

1. Plain X-ray films: microcephaly

2. MRI (Mahmood et al. 2011)

(a) Reduction of cerebral cortical volume (neuronal proliferation defect) and simplification of gyral pattern in most cases

(b) Pachygyria with cortical thickening as well as hypoplasia/agenesis of the corpus callosum (neuronal migration defect) in some cases (ASPM, WER62)

(c) Lissencephaly, schizencephaly, polymicrogyria, and cerebellar hypoplasia in few cases (WDR62)

3. Abnormal cytogenetic findings: high proportion of prophase-like cells due to premature chromosome condensation reported in MCPH1-related MCPH (Jackson et al. 2002; Trimborn et al. 2004, 2005)

4. Molecular genetic analysis (clinical research laboratories listed in www.GeneTests.org)

\section{Genetic Counseling}

1. Recurrence risk

(a) Autosomal recessive inheritance

(i) Patient's sib: $25 \%$

(ii) Patient's offspring: not increased unless the spouse is also a carrier

(b) The empiric recurrence risk for a non-consanguineous couple who have had one child with a diagnosis of MCPH: 1 in 5 if detailed chromosome studies and neuroimaging yield normal results (Tolmie et al. 1987)

(c) There may be a phenotype in heterozygous carriers of MCPH mutations, because of an increased frequency of intellectual impairment in parents of individuals with recessive microcephaly (Kloepfer et al. 1964; Qazi and Reed 1975)

2. Prenatal diagnosis

(a) Ultrasonography: microcephaly

(b) Prenatal MRI studies of affected families: the frontal lobes of the cerebral cortex to be particularly affected (Desir et al. 2008; Saadi et al. 2008)

(c) Molecular genetic analysis: currently available 
3. Management: symptomatic
(a) Early intervention
(b) Speech therapy
(c) Physical therapy
(d) Occupational therapy
(e) Behavior management
(f) Seizure management

\section{References}

Awad, S., Al-Dosari, M. S., Al-Yacoub, N., et al. (2013). Mutation in PHCl implicates chromatin remodeling in primary microcephaly pathogenesis. Human Molecular Genetics, 22, 2200-2213.

Desir, J., Cassart, M., David, P., et al. (2008). Primary microcephaly with ASPM mutation shows simplified cortical gyration with antero-posterior gradient pre- and post-natally. American Journal of Medical Genetics. Part A, 146A, 1439-1443.

Genin, A., Desir, J., Lambert, N., et al. (2012). Kinetochore KMN network gene CASC5 mutated in primary microcephaly. Human Molecular Genetics, 21, 5306-5317.

Hussain, M. S., Baig, S. M., Neumann, S., et al. (2012). A truncating mutation of CEP135 causes primary microcephaly and disturbed centrosomal function. American Journal of Human Genetics, 90, 871-878.

Jackson, A. P., Eastwood, H., Bell, S. M., et al. (2002). Identification of microcephalin, a protein implicated in determining the size of the human brain. American Journal of Human Genetics, 71, 136-142.

Kaindl, A. M., Passemard, S., Kumar, P., et al. (2010). Many roads lead to primary autosomal recessive microcephaly. Progress in Neurobiology, 90, 363-383.

Kloepfer, H. W., Platou, R. V., \& Hansche, W. J. (1964). Manifestations of a recessive gene for microcephaly in a population isolate. Journal de Génétique Humaine, 13, 52-59.

Mahmood, S., Ahmad, W., \& Hassan, M. J. (2011). Autosomal recessive primary microcephaly (MCPH): Clinical manifestations, genetic heterogeneity and mutation continuum. Orphanet Journal of Rare Diseases, 6, 1-15.

Neitzel, H., Neumann, L. M., Schindler, D., et al. (2002). Prematurae chromosome condensation in humans associated with microcephaly and mental retardation: A novel autosomal recessive condition. American Journal of Human Genetics, 70, 1015-1022.

Qazi, Q. H., \& Reed, T. E. (1975). A possible major contribution to mental retardation in the general population by the gene for microcephaly. Clinical Genetics, 7, 85-90.

Saadi, A., et al. (2008). Compound heterozygous ASPM mutations associated with microcephaly and simplified cortical gyration in a consanguineous Algerian family. European Journal of Medical Genetics, 52, 180-184.

Thornton, G. K., \& Woods, C. G. (2009). Primary microcephaly: Do all roads lead to Rome? Trends in Genetics, 25, 501-510.

Tolmie, J. L., McNay, M., \& Stephenson, J. B. P. (1987). Microcephaly: Genetic counselling and antenatal diagnosis after the birth of an affected child. American Journal of Medical Genetics, 27, 583-594.

Trimborn, M., Bell, S. M., Felix, C., et al. (2004). Mutations in microcephalin cause aberrant regulation of chromosome condensation. American Journal of Human Genetics, 75, 261-266. 
Trimborn, M., Richter, R., Sternberg, N., et al. (2005). The first missense alteration in the MCPH1 gene causes autosomal recessive microcephaly with an extremely mild cellular and clinical phenotype. Human Mutation, 26, 496.

Woods, C. G., Bond, J., \& Enard, W. (2005). Autosomal recessive primary microcephaly (MCPH): A review of clinical, molecular, and evolutionary findings. American Journal of Human Genetics, 76, 717-728.

Yang, Y. J., Baltus, A. E., Mathew, R. S., et al. (2012). Microcephaly gene links trithorax and REST/ NRSF to control neural stem cell proliferation and differentiation. Cell, 151, 1097-1112. 